\title{
Changes in Cardiac Rate and Rhythm Produced by Electrical Stimulation of the Brain Stem of Dogs
}

\author{
Hideo UEDA, M.D., ${ }^{*}$ Tsuneaki SUGIMOTO, M. D., Satoru MURAO, \\ M. D., Hitoshi GOTO, M.D., Kazuzo KATO, M. D., Soichi \\ KATAYAMA, M.D., and Kiyoshi ITO, M.D.
}

The changes in cardiac rate and rhythm induced by the electrical stimulation on the brain stem of dogs were studied. The stimulation on the posterior part of the hypothalamus, the midline, ventral and medial nuclei groups in the thalamus and the posterior commissure, the central gray stratum and a part of reticular formation in the midbrain mainly produced a marked sinus tachycardia. However, in the late phase or after the end of stimulation, there often occurred sinus bradycardia accompanied by atrioventricular block or nodal escaped beat. This phenomenon was explained to be due to delayed vagal hyperactivity. The ventricular extrasystoles which most frequently occurred on stimulation of the brain stem were classified into 2 groups according to the mode of appearance. The investigation on the electrocardiographic features of those of 2 types, the time of their appearance, their relation to the changes in blood pressure and the phase of respiration suggested that the one was due to direct neural excitation of cardiac ectopic pacemaker, while the other was of secondary nature being associated with blood pressure elevation. The central nervous representation of such extrasystole as of former type was found to be relatively restricted area in the median part of the brain stem.

$\mathrm{T}$ $\mathrm{HE}$ changes in heart rate or cardiac arrhythmias are often precipitated by functional or organic stress on the central nervous system, emotional excitement, cerebral hemorrhage, head injury or transcranial electroshock and so on. This relationship between the central nervous system and the cardiac arrhythmias has been noticed for a long time, and there are also a great deal of experimental evidence on this problem under widely varying conditions.

From the Second Department of Internal Medicine, Faculty of Medicine, University of Tokyo, Tokyo.

*Professor of Internal Medicine. 
Levy $(1912)^{1)}$ was the first one who suggested the participation of the central nervous system in producing cardiac arrhythmias in cats under chloroform anesthesia. Further progress was made by the detailed investigations of Beattie, Brow and Long (1930).2) They found that a center existed in certain region in the brain stem, the removal or stimulation of which resulted in abolition or production of extrasystoles. Since then, many studies, using electrical stimulation on the brain stem, or by injection of drugs into the cerebral ventricles, have been published, such as of Allen (1932), Dikshit (1934), ${ }^{3)}$ van Bogaert (1936), Korth et al. $(1937),{ }^{6)}$ Shinozaki et al. (1939, 1940, 1941), ${ }^{7}$. Weinberg et al. (1955, 1956, $1950),{ }^{8}$ Korteweg et al. $\left.(1957),{ }^{9}\right)$ Yuasa et al. $(1957)^{10)}$ and Purpura et al. (1958). ${ }^{11)}$

However, in these experimental studies, it was not clearly demonstrated whether specific regions in the central nervous system were responsible for the production of arrhythmias, and whether thus centrally induced arrhythmias were caused by direct neural excitation of the heart and/or by concomitant changes in blood pressure, respiration and convulsion. In this paper, authors tried to clarify these problems.

\section{Method}

Fifty-one dogs were anesthetized with morphine chloride alone $(6 \mathrm{mg} . / \mathrm{Kg}$.), or the combination of morphine chloride $(1-3 \mathrm{mg} . / \mathrm{Kg}$.) with chloralose $(50-70$ $\mathrm{mg} . / \mathrm{Kg}$.). Using the stereotaxic instrument, the bipolar concentric stimulation electrode was inserted. The location of the tip of this electrode was determined by histological examination after each experiment. The electrical stimuli were rectangular voltage pulses; their duration was $1 \mathrm{msec}$; their frequency 100 cycle per second; and their voltage 1-10 volts (4-30 volts in crest value). Each stimulus was applied for 15-30 seconds.

Electrocardiogram was taken on the standard limb leads I, III and the intraatrial lead. The blood pressure was recorded on a kymograph by means of a mercury manometer connected to the femoral artery. The respiratory movement of the thorax was registered on a kymograph by means of a tambour connected to the cuff wrapping around the thorax, and recorded also on the paper of electrocardiogram simultaneously using a transducer of rubber tube filled with zinc sulfate solution.

The dogs with blood pressure under $100 \mathrm{~mm}$. Hg or heart rate over 180 beats per minute were excluded from the study.

\section{Results}

In the present study the stimulation was given to 87 points in the thalamus, 51 points in the hypothalamus and 104 points in the midbrain and adjacent area. 
1. Changes in heart rate

The changes in heart rate are shown in Table I-III. The grade of changes is expressed as a ratio of the heart rates for each 5 sec. before and during the stimulation. Fig. 1 is the map of the brain stem which shows the distribution of the stimulated points. In our experimental conditions, cardiac acceleration was observed in most of the cases, and cardiac inhibition was rarely encountered.

In the thalamus, moderate tachycardia indicating over $100 \%$ increase of the basal rate was observed mainly when the stimulated points were in the midline, ventral and medial nuclei groups. On the stimulation of other nuclei groups, cardiac acceleration was in a lesser degree.

In about $30 \%$ of all experiments on hypothalamic stimulation, there also appeared moderate tachycardia. Mild cardiac inhibitory responses were obtained by the stimulation of some parts of preoptic region and anterior and medial hypothalamus.

In the midbrain and the adjacent area, the stimulation of the central gray stratum, the posterior commissure, the reticular formation, the quadrigeminal bodies and the medial lemniscus produced mostly tachycardiac responses, while bradycardia was infrequently observed.

On the stimulation of each points, cardiac acceleration was usually accompanied by the elevation of blood pressure. But, as shown in Fig. 2, these did not always occur in parallel with each other.

Generally, the acceleration of the heart rate was maximum in the early phase of the stimulation, and in the later phase of the stimulation the heart rate almost returned to the basal level. However in some cases, as shown in Fig. 2, there developed a marked sinus bradycardia associated with atrioventricular block, nodal escaped beat or nodal escaped rhythm in the later phase or after cessation of the stimulation. As a rule such a response was accompanied by a marked elevation of blood pressure, but there were some points in which delayed vagal hyperactivity occurred with even slight elcvation of the blood pressure $(<40 \%)$. Most of these points were distributed in the anterior and medial part, a few in the posterior part of the hypothalamus and in the medial nuclei group in the thalamus.

\section{Conduction disturbances}

Immediately after the onset of the stimulation there occurred no conduction disturbance. When the conduction disturbance was elicited, it usually occurred in the late phase of the stimulation or immediately following the stimulation with development of delayed vagal hyperactivity as described above. They always appeared as atrioventricular block with first or second degree.

\section{Ectopic arrhythmias}




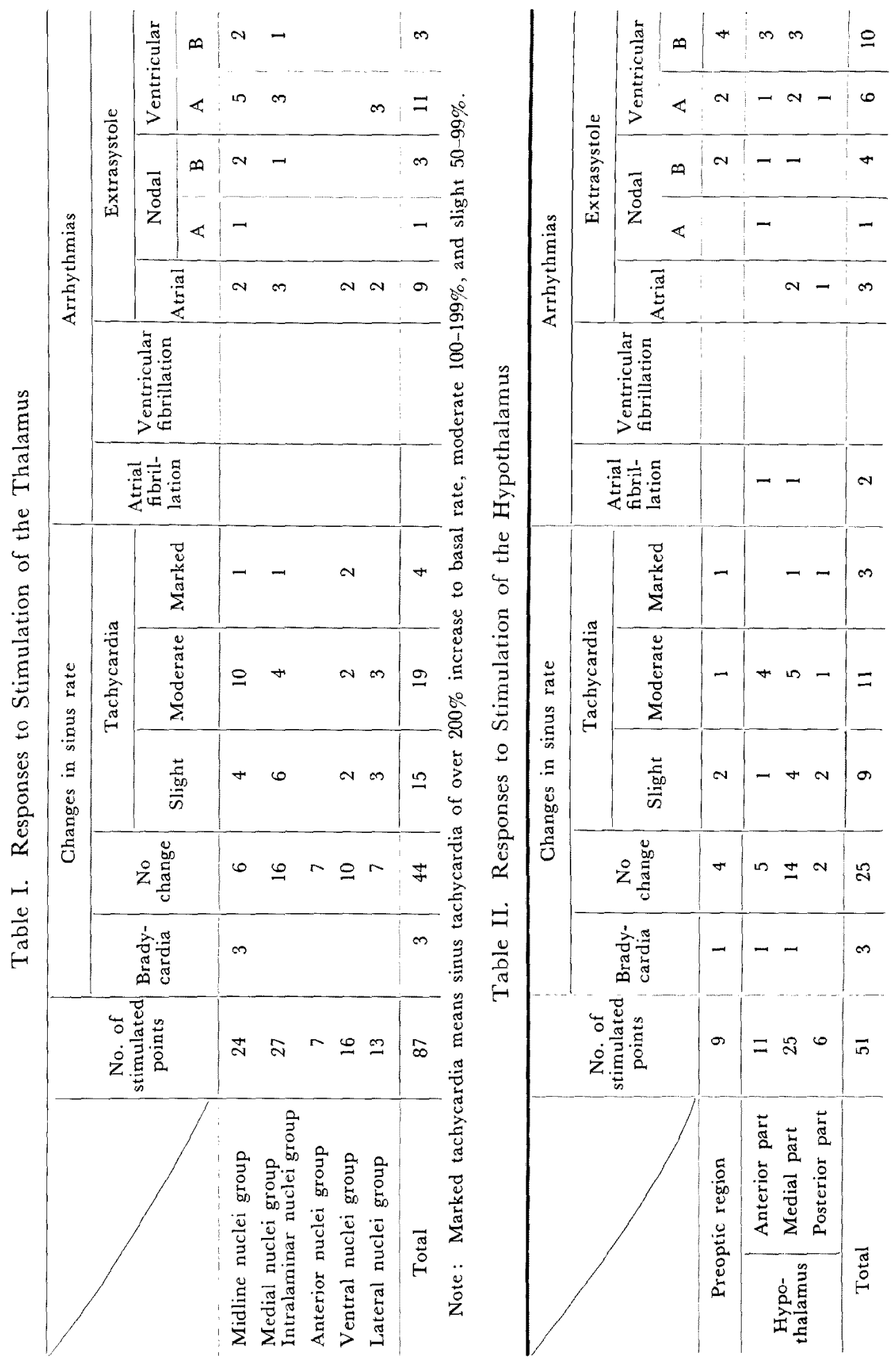




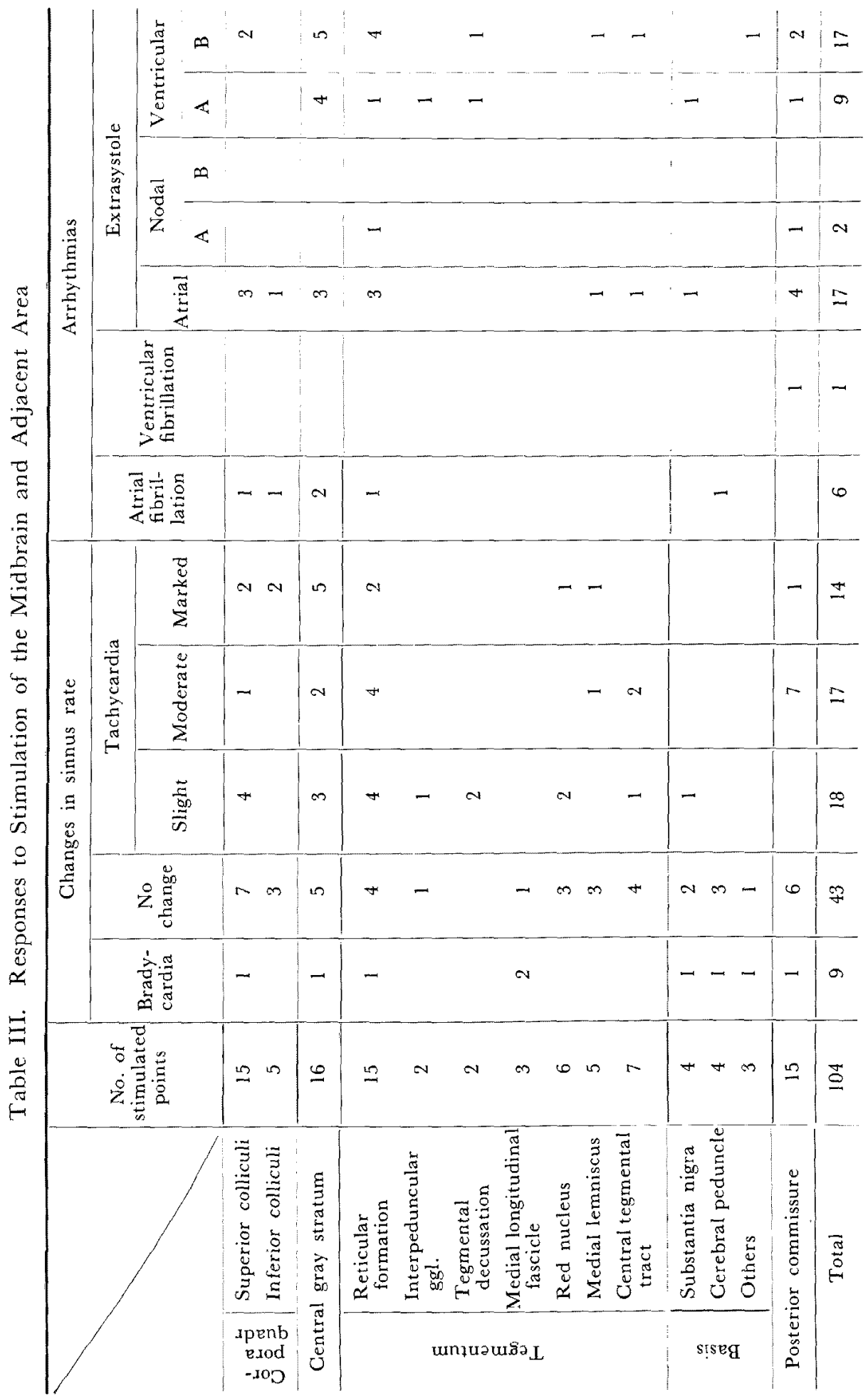




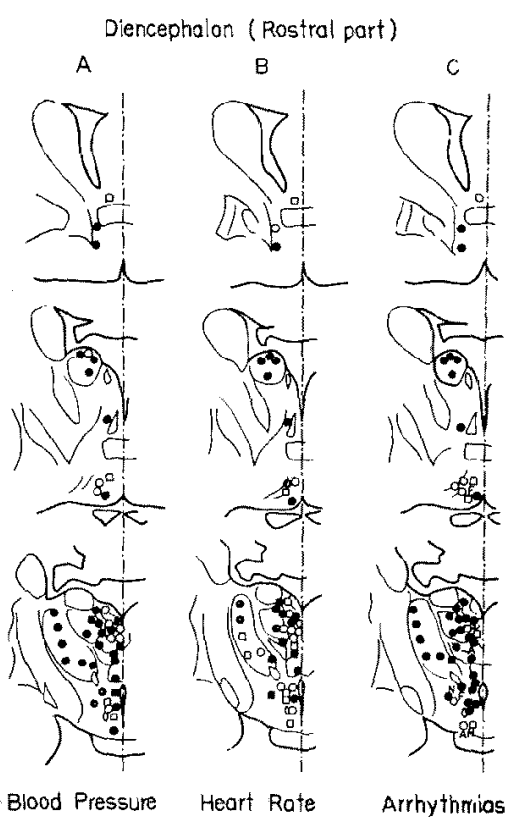

(a)

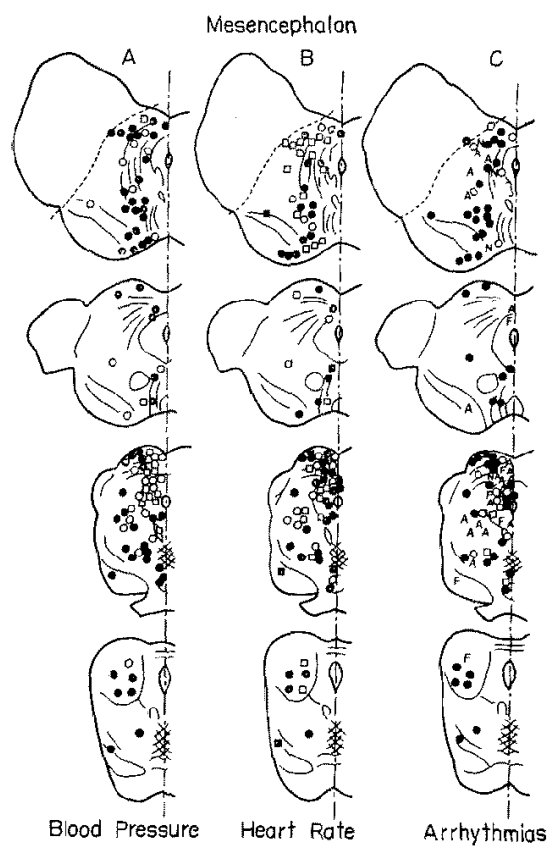

(c)

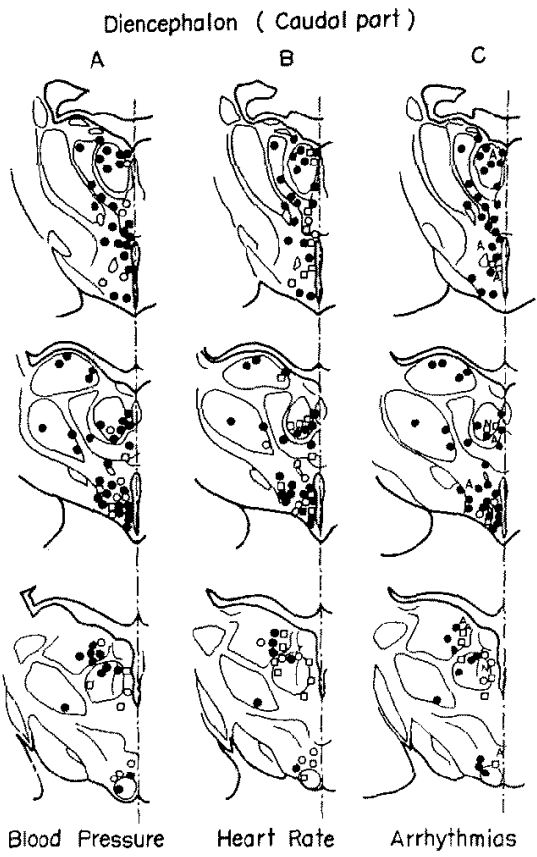

(b)

Fig. 1a. Drawings of frontal section of the brain stem of the dog, showing the location of stimulated points. Vasomotor effects on the right (A), changes in heart rate on the middle (B), and types of arrhythmias on the left side (C).

Marks are as follows:

Blood pressure responses

[- Marked elevation (over $80 \%$ increase to control level)

Moderate elevation (40-79\%)

- Slight or no change $(0-39 \%)$ Fall

Changes in heart rate

1. Marked sinus tachycardia (over $200 \%$ increase to basal rate)

$\bigcirc$ Moderate sinus tachycardia (over $100 \%$ increase)

- Slight or no change

- Sinus bradycardia

Types of arrhythmias

$\square$ Ventricular extrasystole of type ' $A$ ',

Ventricular extrasystole of typc ' $B$ '

N Nodal extrasystole

A Atrial extrasystole

F Atrial fibrillation

- No arrhythmias

Fig. 16, c. Symbols are the same to those in Fig. Ia. 


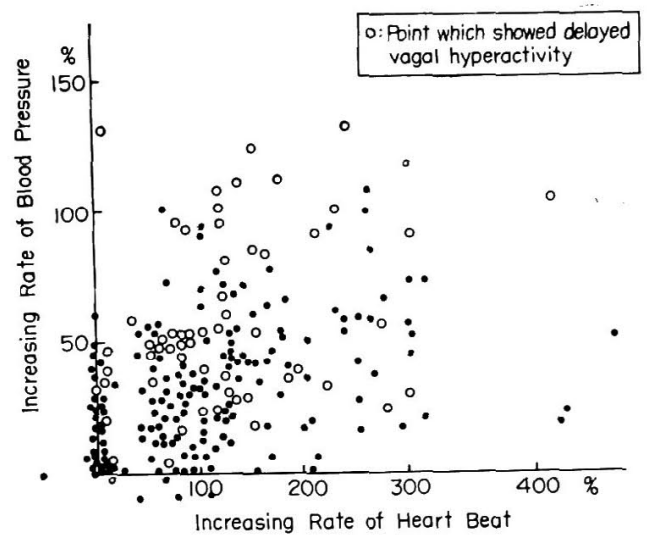

Fig. 2. The relationship between blood pressure response and changes in heart rate.

By the stimulation of the brain stem, various arrhythmias, such as atrial extrasystole, atrial fibrillation, nodal extrasystole, ventricular extrasystole, ventricular tachycardia and ventricular fibrillation etc. were obtained (Fig. 3-7). Their incidence and the distribution of the eliciting points are shown in Table I-III and Fig. 1. In these tables and figure, escaped beat with ventricular rate under preexciting sinus rate was excluded.

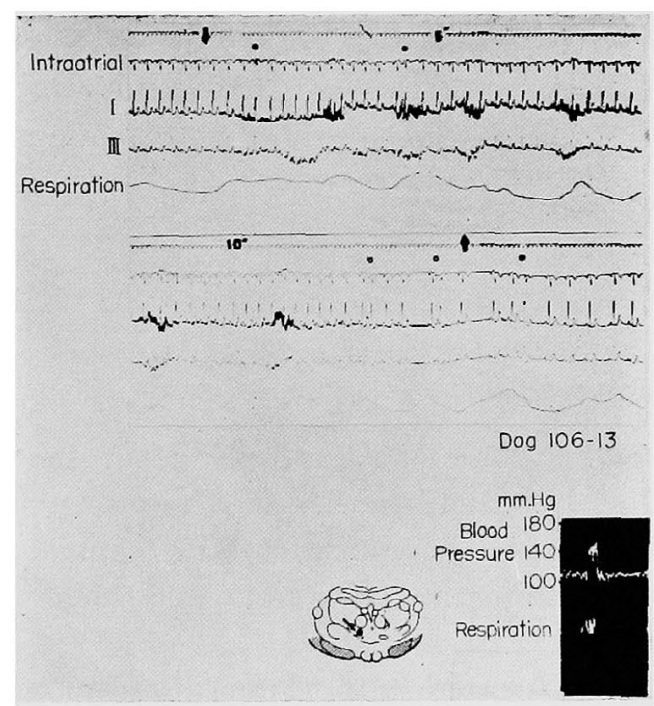

Fig. 3. Ventricular and atrial extrasystoles produced by stimulation of the thalamus (nucl. commissuralis interventralis). 'The extrasystoles marked with $\bullet$ are ventricular extrasystole and those marked with $O$ atrial ones. 


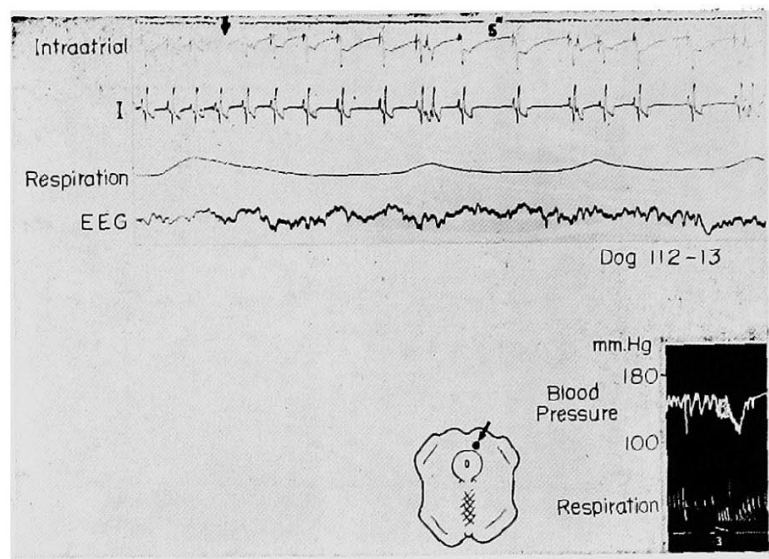

Fig. 4. Atrial extrasystoles produced by stimulation of the midbrain (commissure superior colliculi).

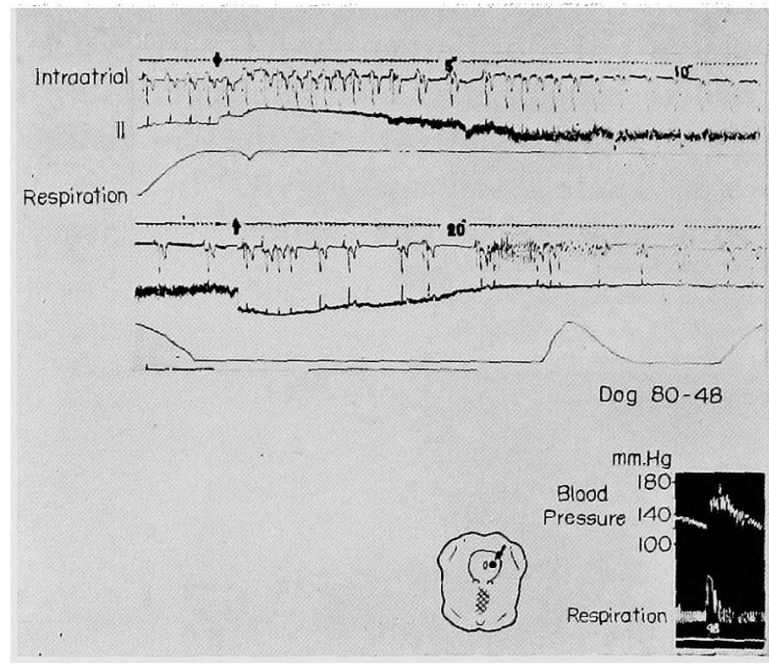

Fig. 5. Atrial fibrillation produced by stimulation of the central gray stratum.

Atrial extrasystoles were often observed on the stimulation of the posterior and medial parts of the hypothalamus, most nuclei groups in the thalamus, the posterior commissure, the central gray stratum, the quadrigeminal bodies, the substantia nigra and some part of the tegment of the midbrain.

Atrial fibrillation appeared in the later phase or after the cessation of the stimulation, and it was often preceded by a marked elevation of blood pressure and atrial extrasystoles. It was observed on the stimulation of the anterior and medial parts of the hypothalamus, the quadrigeminal bodies, the central gray stratum, the reticular formation and the cercbral 


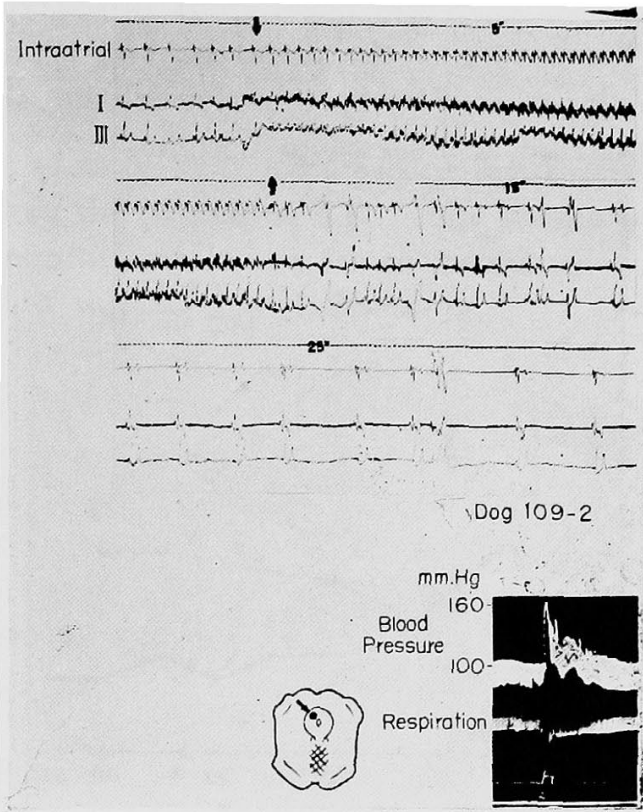

Fig. 6. Ventricular extrasystoles produced by stimulation of the central gray stratum.

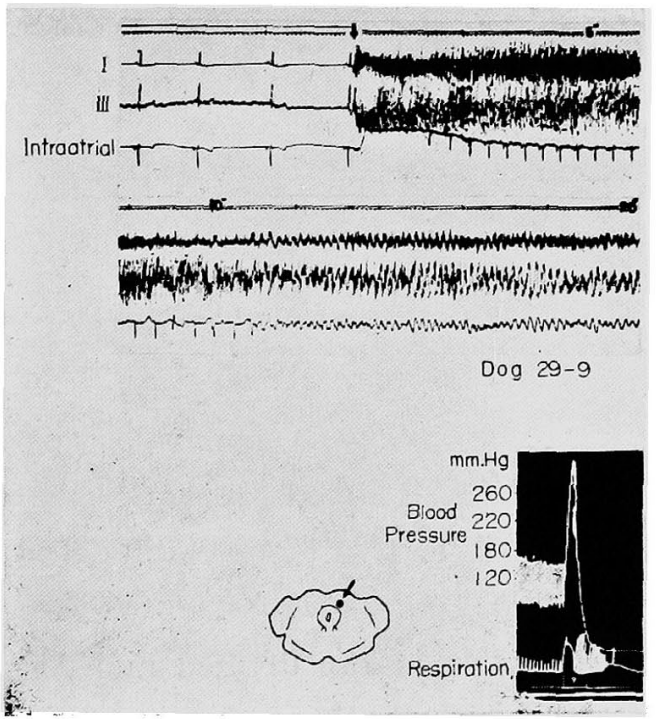

Fig. 7, Ventricular fibrillation produced by stimulation of the posterior commissure.

peduncle in the midbrain.

Nodal extrasystole was classified into 2 groups, one was apparently premature extrasystole (type 'A'), the other was an escaped beat of 
coupling interval shorter than the basal sinus rate (type ' $\mathrm{B}$ '). It seems difficult to decide the nature of the nodal beat of type ' $B$ ' to be of passive or active. As shown in Fig. 8, the slower the sinus rate becomes, the slower the nodal rate also seems to become.

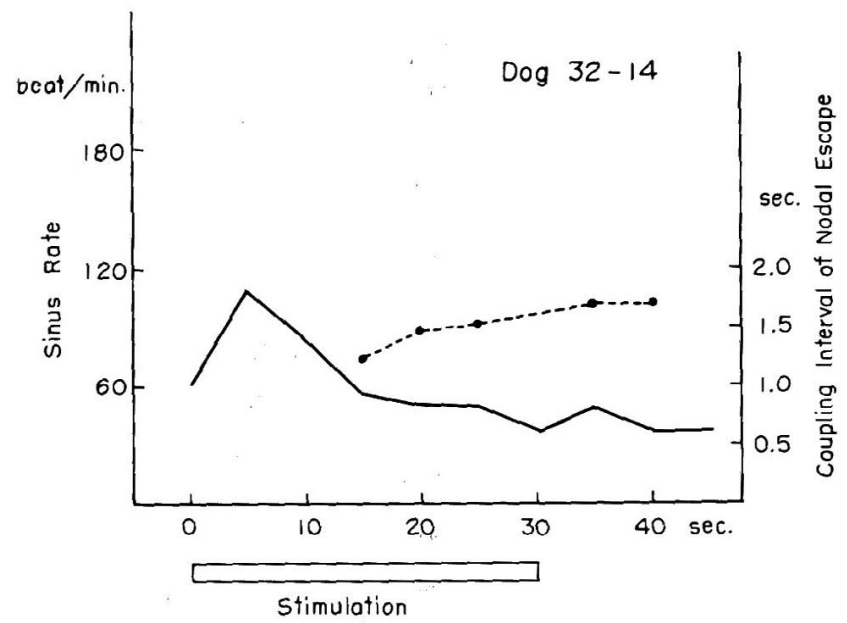

Fig. 8. The relationship between sinus rate and the coupling interval of nodal escaped beats on hypothalamic stimulation. The solid line shows the change of sinus rate, and the dotted line the change of coupling interval.

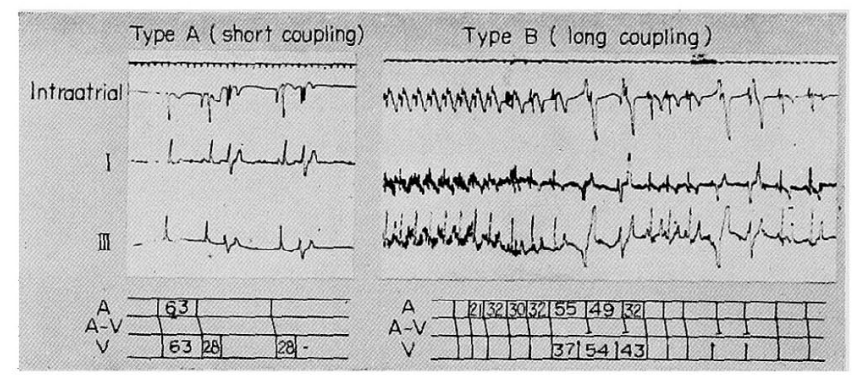

Fig. 9. Two types of ventricular extrasystoles.

4. Ventricular extrasystole

Ventricular extrasystole was also divided into 2 groups, ' $A$ ' and 'B', according to the mode of appearance (Fig. 9). Extrasystole of type 'A' was a premature ventricular contraction of shorter coupling interval (usually $0.20-0.30 \mathrm{sec}$ ) and frequently appeared in the early phase of stimulation. The mode of appearance of extrasystole of type ' $B$ ' somewhat simulated to that of escaped beat, because it was of longer coupling interval (usually more than $0.30 \mathrm{sec}$.) and seemed to be precipitated by 
vagal inhibition of sinus rate in the later phase or after the cessation of the stimulation. Fig. 10 shows the relation between the coupling interval and preceding $R R$ of these 2 types of ventricular extrasystoles. The coupling interval of type ' $\mathrm{A}$ ' seemed to be distributed near the standard QT curve referring to each ventricular cycle, while that of type ' $B$ ' was frequently longer than the preceding RR. The former sometimes occurred as bigeminy or trigeminy, while the latter frequently occurred as ventricular tachycardia with interference dissociation.

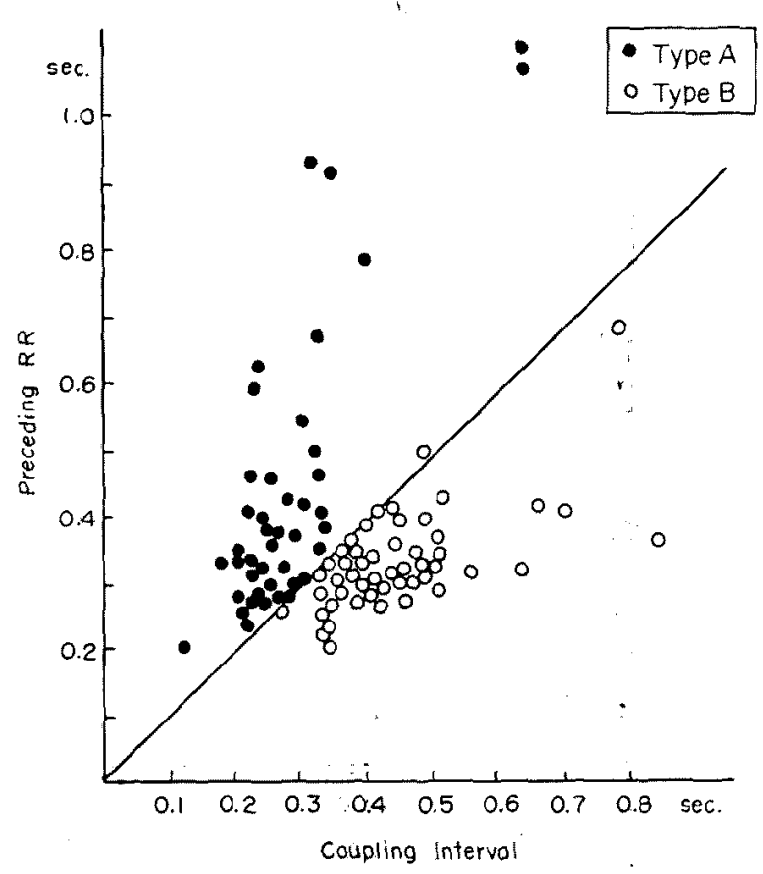

Fig. 10. Preceding RR and coupling interval of ventricular extrasystoles.

The characteristics of these 2 types was found in the relation with the change in blood pressure (Fig. 11). While the extrasystole of type 'A' tended to appear independently to the change in blood pressure, the incidence of extrasystole of type ' $B$ ' was closely related to the degree of blood pressure elevation. In 29 out of 46 experiments which showed a marked elevation of blood pressure of over $80 \%$, extrasystoles of type ' $B$ ' were observed.

In relation to the phase of respiration, it was found that the extrasystole of type 'A' tended to occur in the inspiratory phase, on the other hand, the one of type ' $\mathrm{B}$ ' in the expiratory phase. Physiologically RR and/ or PR prolongation occurs in expiratory phase, and this would precipitate the occurrence of extrasystole of type ' $B$ '. 


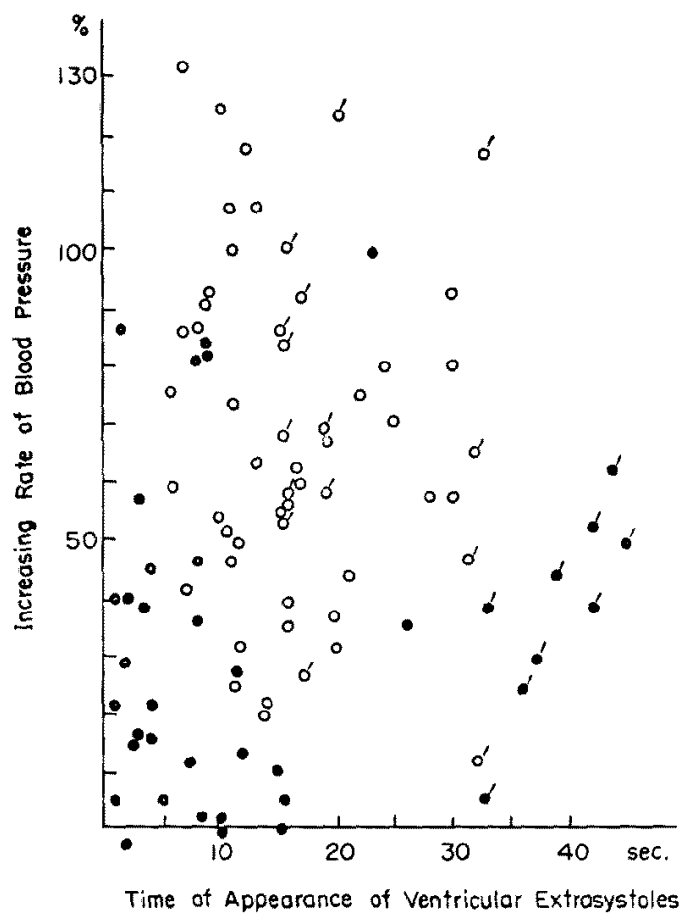

Fig. 11. Changes in blood pressure and the time of appearance of ventricular extrasystoles of two types.

- : type 'A', $O$ : type 'B', ': extrasystoles which appeared for the first time after stimulation had ceased.

Moreover the shape of ventricular complex in these 2 types tends to have some specificity. In the present experimental study, ventricular extrasystoles could be classified into \& types according to the direction of the main deflection of QRS complex of I, III and intraatrial lead (Table IV). As

Table IV. Four Types of Ventricular Extrasystoles

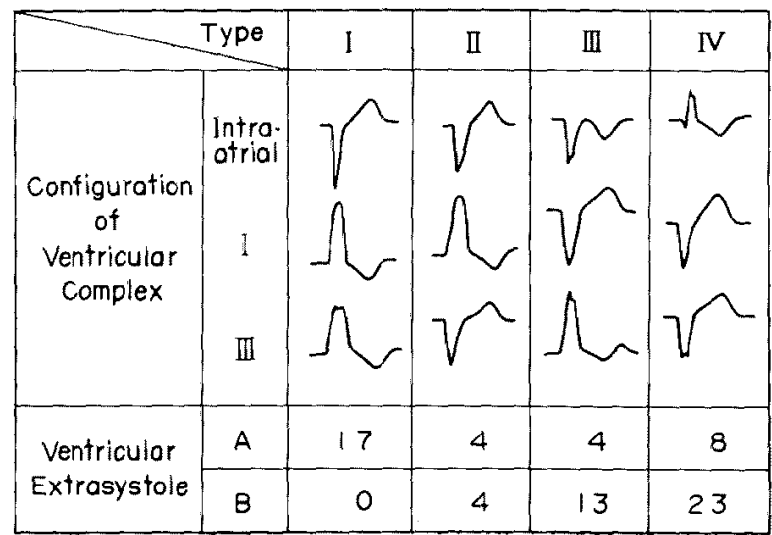


shown in Table IV, the extrasystole of type ' $A$ ' is mostly of type I and the one of type ' $\mathrm{B}$ ' is of type IV. Type I may have its ectopic focus in the basal portion of right ventricle and type IV in the apical portion of left ventricle. This is an interesting finding, considering that extrasystole of type ' $B$ ' may be due to secondary effects such as blood pressure elevation.

Since the extrasystole of these 2 types seemed to be of different mechanism, in Fig. 1, points which produced ventricular extrasystole of type 'A' or 'B' were plotted separately on the map. The points responsible for extrasystole of type 'A' seemed to be rather concentrated in the median parts of the brain stem especially near by the third ventricle and aquaeductus-the midline, and medial nuclei groups in the thalamus, the median part of the hypothalamus and the central gray stratum of the midbrain. On the other hand, the points for type ' $\mathrm{B}$ ' were scattered in relatively widespread regions associated with marked rise of blood pressure.

5. Secondary factors which may influence the production of arrhythmias

1) Vagus nerve

Participation of vagal element in the production of arrhythmias could be demonstrated by the following procedures. Generally, after atropinization or section of bilateral vagi, there occurred no more vagal slowing of sinus rate in later phase or even after the cessation of the stimulation as observed before. And the occurrence of extrasystole was also diminished. After vagotomy the blood pressure did not return so abruptly to its original level as in the non-vagotomized animal.

The following example may serve to illustrate the role of vagus nerve (Fig. 12, 13); in this experiment, the stimulation of the central gray stratum in the midbrain caused ventricular extrasystoles $13.5 \mathrm{sec}$. after the onset of the stimulation, but if vagal stimulation was added in the midst of mesencephalic stimulation and sinus rate was depressed, ventricular extrasystoles appeared earlier. Then vagi were cut bilaterally and the stimulation was repeated with the same condition. During the stimulation there were no arrhythmias of any kinds, but when vagal stimulation was added, there occurred various extrasystoles accompanying with sinus depression, which disappeared again after the cessation of vagal stimulation.

2) Blood pressure

Table $\mathrm{V}$ shows that the incidence of arrhythmias, particularly ventricular extrasystole of type ' $B$ ', increases with the grade of blood pressure elevation. It is considered that the blood pressure elevation itself and/or its associated responses such as liberation of catecholamines act on the production of arrhythmias directly or secondarily via reflex 


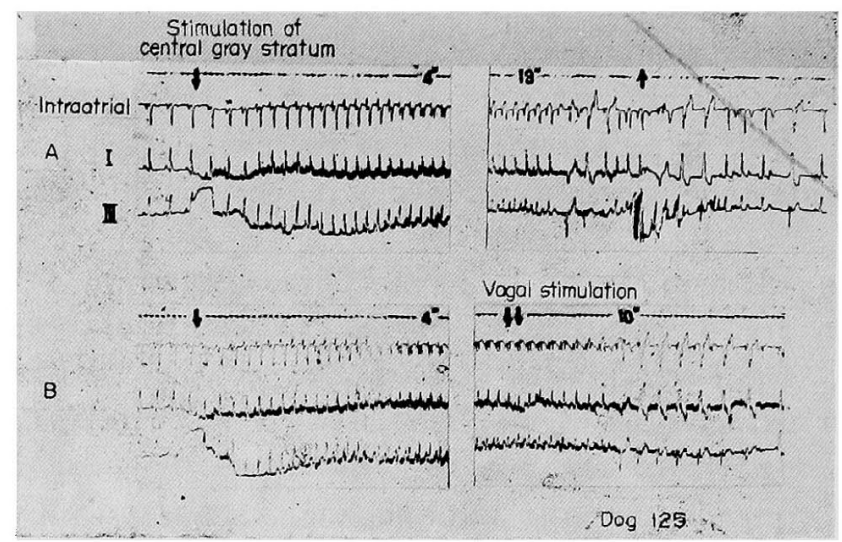

Fig. 12. The effect of superimposed vagal stimulation. The vagus nerves were intact.

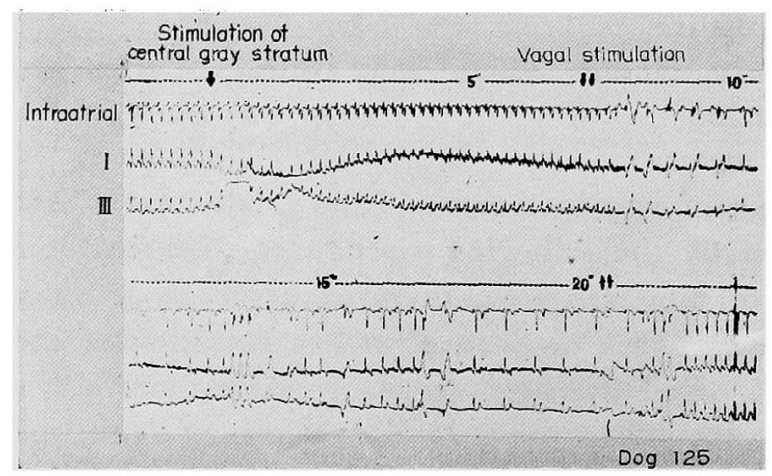

Fig. 13. The effect of added vagal stimulation, after the section of the bilateral vagi.

Table V. The Relation between Blood Pressure Changes and Various Arrhythmias

\begin{tabular}{|c|c|c|c|c|c|c|c|}
\hline & & & \multicolumn{4}{|c|}{ Increasing rate of pressure } & \multirow{2}{*}{ Total } \\
\hline & \multicolumn{2}{|l|}{. } & $\sim 0$ & $0 \sim 39$ & $40 \sim 79$ & $80 \sim \%$ & \\
\hline \multicolumn{3}{|c|}{ No. of experiments } & 19 & 354 & 136 & 46 & 555 \\
\hline \multicolumn{3}{|c|}{$\begin{array}{l}\text { Atrial fibrillation } \\
\text { Ventricular fibrillation }\end{array}$} & 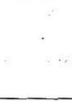 & 3 & 5 & $\begin{array}{l}3 \\
1\end{array}$ & $\begin{array}{r}11 \\
1\end{array}$ \\
\hline \multirow{3}{*}{ Extrasystole } & Atrial & & 1 & 26 & 29 & 4 & 60 \\
\hline & Nodal & $\begin{array}{l}\text { A } \\
\text { B }\end{array}$ & . & $\begin{array}{l}2 \\
4\end{array}$ & $\begin{array}{r}2 \\
10 \\
\end{array}$ & $\begin{array}{l}2 \\
4\end{array}$ & $\begin{array}{r}5 \\
18\end{array}$ \\
\hline & Ventricular & $\begin{array}{l}\mathrm{A} \\
\mathrm{B}\end{array}$ & 3 & $\begin{array}{r}24 \\
6\end{array}$ & $\begin{array}{l}13 \\
32\end{array}$ & $\begin{array}{r}7 \\
\quad \quad 29\end{array}$ & $\begin{array}{l}47 \\
67\end{array}$ \\
\hline
\end{tabular}


mechanism.

3) ST-T change

Stimulation of the brain stem also frequently induced other electrocardiographic changes, especially ST depression and flattening or inversion of $\mathrm{T}$ wave. In general, ST-T change was distinct when the rise of blood pressure was marked, and it occurred gradually with latency of a few or several seconds and lasted after the cessation of the stimulation. There was no distinct relation between the incidence of ST-T change and arrhythmias.

4) Convulsion

Autonomic responses induced by central nervous stimulation were often accompanied by tonic or clonic convulsion. To study the influence of convulsion on the production of arrhythmias, some experiments were performed on the immobilized animals. Fig. 14 shows an experiment

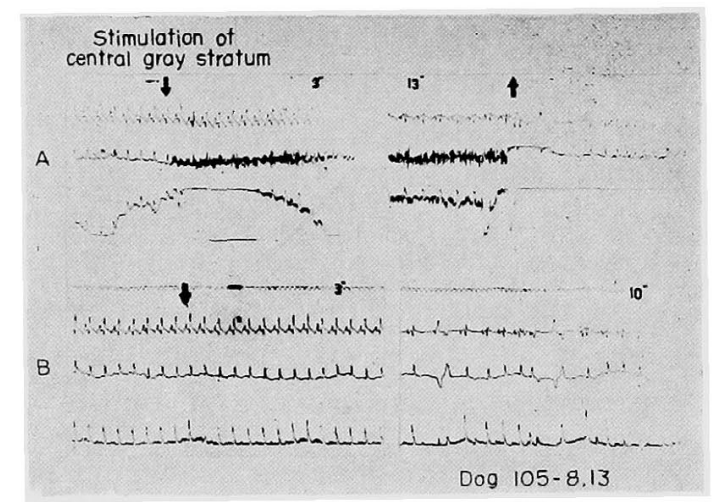

Fig. 14. The influence of convulsion on the production of arrhythmias. A: before the immobilization, B: after intravenous injection of succinyl-choline.

which demonstrated that even after intravenous injection of succinyl choline, the hypothalamic stimulation still produced ventricular extrasystoles of the same type as before.

\section{Discussion}

1. Ghanges in heart rate

Numerous studies have demonstrated that the hypothalamus are richly endowed with areas which yield marked vasomotor effects upon electrical excitation. In the detailed studies of Karplus and Kreidl,12) Hess, ${ }^{13)}$ and Kabat, Magoun and Ranson, ${ }^{14)}$ the anatomical sites concerned with vasomotor responses in the hypothalamus have been described precisely. In general, vasopressor responses were found to be most 
common in the lateral and posterior hypothalamic regions, whereas vasodepressor points were more commonly encountered anteriorly and medially in the hypothalamus. However, there is considerable overlapping of the areas giving these opposing responses.

On the changes in the heart rate produced by hypothalamic stimulation, there have been also many observations. Wang and Ranson (1941), ${ }^{15)}$ in cats under chloralose anesthesia, observed bradycardia on stimulation of the preoptic region and tachycardia on stimulation of the hypothalamus. According to van Bogaert (1936), ${ }^{5)}$ Weinberg et al. $(1960)^{8)}$ and Bruno et al. (1961), ${ }^{16)}$ those tachycardiac responses are most easily obtained from the posterior or posterolateral hypothalamic region. Korteweg et al. $(1957)^{9)}$ described that the anterior part of the hypothalamus produced bradycardia, while the posterior part produced no change during the stimulation and bradycardia or tachycardia after the stimulation had ceased. Further, Kurotsu et al. (1951), ${ }^{10)}$ who divided the hypothalamus into 3 zones, ' $a$ ', ' $b$ ' and ' $c$ ', in unanesthetized rabbits, showed that ' $b$ ' sympathetic zone produced tachycardia and ' $c$ ' parasympathetic zone had the effect of bradycardia after the cessation of stimulation.

In our experiments, sinus tachycardia was much more frequently observed than bradycardia as a result of the stimulation of the preoptic region or the hypothalamus. Although the reason of these result may be due to the difference of the experimental animals, anesthetic drugs or the methods of stimulation, it will be mostly responsible that the animals of high vagal tone were used as a consequence of opium anesthesia. Since the preexisting autonomic state of the animal inclined to vagal, the parasympathetic response might be scarcely obtained. However, the points which showed delayed bradycardia response without a marked elevation of blood pressure were found mainly in the anterior and medial parts of the hypothalamus. The delayed bradycardia response may be partly due to the effect of the stimulation of the parasympathetic areas which was masked by sympathetic reaction in the early phase of the stimulation.

There are few systematic studies on the autonomic function of the thalamus. Ueda et al. $\left.(1960)^{17}\right)$ reported that marked cardiovascular responses were obscrved on the stimulation of the midline and medial nuclei groups and slight or little in the other nuclei groups. And, considering the anatomical relationship between the thalamic nuclei and the higher autonomic areas, they concluded that the autonomic representation located in the medial part of the thalamus. As to the change in the heart rate, Monnier (1955) ${ }^{18}$ ) observed cardiac acceleration on the stimulation of 'région médiane', but Korteweg et al. $(1957)^{9)}$ obtained no changes by thalamic stimulation in cats. 
The midbrain is said to serve as a route for fibers of descending pathway which are concerned with cardiovascular activities. In our experiments, a marked cardiac acceleration was observed mainly on the stimulation of the central gray stratum, a part of the reticular formation, the medial lemniscus, the quadrigeminal bodies and the posterior commissure. This fact is in accordance with the anatomical findings that the hypothalamic-medullary efferents have a somewhat diffuse extension, partly passing the superior colliculi, in the lateral portions of the tegmentum and in the central gray stratum and also in the medial longitudinal bundle and the medial reticular formation. ${ }^{25)}$ The brain stem reticular formation system must be viewed not only as pathway of the hypothalamic impulse but also as the site of integration of a wide variety of afferent drives capable of affecting vagosympathetic centers controlling cardiovascular system.

2. Delayed vagal activity

Tachycardia was sometimes followed by a marked bradycardia in the later phase of the stimulation or soon after the stimulation had ceased. One would expect such bradycardia response presumably due to secondary vagal reflex or the effect of the stimulation of parasympathetic area which had been masked by sympathetic reaction. It is interesting to note, in connection with the latter mechanism, the fact that some of those points showing delayed vagal hyperactivity without remarkable rise of blood pressure were found in the area which had been described as parasympathetic.

Atrioventricular block, nodal escaped beat or nodal escaped rhythm with interference dissociation were often seen in the late or poststimulation period. Those are thought to be secondary phenomenon of vagal hyperactivity above mentioned. This fact was also observed by Korteweg et al. and Weinberg et al. Weinberg et al. $(1960)^{8)}$ classified the electrocardiographic manifestations as initial and secondary, according to the time of appearance after the onset of diencephalic stimulation. They considered that the secondary effects, such as relative bradycardia, rhythm grouping and pacemaker shifts, were probably the result of delayed reflex activation of accessory cardioregulatory centers within the nervous system.

3. Ventricular ectopic arrhythmias

Many investigators have observed various arrhythmias on electrical or chemical stimulation of the brain stem. Beattie et al. $(1930)^{2)}$ showed that the stimulation of the posterior part of the lateral wall of the third ventricle produced an extrasystolic cardiac arrhythmia in cats under chloroform anesthesia. Allen $(1932)^{3}$ ) confirmed these results and also obtained extrasystolic arrhythmia by the stimulation of the superior 
colliculi of the midbrain of the rabbit anesthetized with sodium barbiturate and ether. Van Bogaert (1936), ${ }^{5)}$ using cats under anesthesia of morphine and chloral hydrate, observed extrasystoles with change in the heart rate during the stimulation of the posterior hypothalamus. By injecting strophantin, acetylcholine, caffein and nicotine into the lateral or third ventricle of cats or dogs, Dikshit (1934),") Korth et al. (1937) ${ }^{6}$ ) and Shinozaki et al. $(1939,1940,1941)^{3)}$ succeeded in producing extrasystoles. They observed that these arrhythmias were usually associated with an elevated blood pressure and often disappeared after the addition of barbiturate anesthesia. Shinozaki et al. described that these arrhythmias were, if not always, prevented by vagotomy but not influenced by the resection of cardiac sympathetics. Korteweg et al., in cats under anesthesia of pentobarbital and ether, obtained cardiac arrhythmias of various kind by the stimulation of the hypothalamus and the quadrigeminal bodies. They also showed that a remarkable rise of blood pressure might be an important factor in the production of arrhythmias appearing after the stimulation had ceased. They differentiated poststimulation alterations from those occurring during stimulation. The former one was abolished by vagotomy whereas the latter was unaffected or made more frequent. The former was considered to be related to blood pressure elevation, mediating via vagus. These results were entirely confirmed in the investigation of Purpura et al. $(1958)^{11)}$ on cats anesthetized with ether.

Authors classified the ventricular extrasystoles into 2 types, 'A', and ' $B$ '. The extrasystole of type ' $A$ ' occurred in the early phase of stimulation and was not necessarily associated with blood pressure elevation. These facts suggest that it is caused by direct effect of central nervous excitation. On the other hand, most of extrasystoles of type ' $B$ ' were accompanied by a marked elevation of blood pressure and occurred often after or in the later phase of the stimulation. Since it resembled escaped beat in its appearance and occurs sometimes as ventricular tachycardia, the extrasystole of type ' $\mathrm{B}$ ' seems to be due to the mechanism that the lower ventricular pacemaker with an increased automaticity takes over the cardiac rhythm, precipitated by vagal inhibition of sinus pacemaker. From the fact that it is of left ventricular origin, associated with an elevated blood pressure it will be possibly considered that the increased automaticity is due to passive ventricular distension owing to blood pressure elevation.

Rothberger and Winterberg $(1910)^{19)}$ and Levy (1914)20) observed ventricular extrasystoles by mechanical elevation of blood pressure, though such a mechanical passive distension of ventricular muscle does not always produce ventricular extrasystoles.

On the other hand, there are many descriptions that the secretion of 
catecholamines increases on the stimulation of the brain stem. In addition, it has been demonstrated that the higher the level of blood pressure, the lesser epinephrine produces ventricular extrasystoles or ventricular tachycardia. ${ }^{21)}$

Probably, both epinephrine released into blood and high blood pressure sustained by central nervous excitation act in cooperation with each other, to produce ventricular extrasystole such as of type 'B'. Moreover, recently we found much epinephrine, enough to sustain high blood pressure level or to produce ventricular extrasystole, was released by central nervous stimulation. This will be reported in another paper.

Another factor which precipitates ventricular extrasystole is suppression of sinus pacemaker. This may be reflex vagal activation due to blood pressure elevation or increased catecholamines, expiratory bradycardia and in some cases, delayed vagal hyperactivity because of stimulation of the parasympathetic area. Central nervous stimulation after vagotomy or adequate atropinization induced only cardiac acceleration and there appeared no more vagal inhibition and cardiac arrhythmias. If, in such a case, vagal stimulation was added and thus depressed sinus rate, the lower ectopic center with increased automaticity took over the whole heart. This experiment indicates that vagal inhibition is an important precipitating factor.

Thus, those extrasystolic arrhythmias which were described by the previous workers, may be analyzed according to their mechanisms of production. Those which were said to appear after the end of the stimulation, accompanied by an elevated blood pressure or associated with bradycardia and those which were said not to be influenced by the section of cardiac sympathetics but to disappear after vagotomy, are probably the ventricular extrasystoles of type 'B'. Those which were described to appear early during the stimulation or independently to the changes in blood pressure may be probably the ventricular extrasystoles of type ' $\mathrm{A}$, in our classification.

The localization responsible for the extrasystole of type ' $A$ ' distributed relatively in the median portion of the brain stem. It will be suggested that these areas have some specific function to influence the heart to produce ventricular extrasystoles.

4. Other factors concerned with arrhythmias

Convulsion has been regarded as one of precipitating factors of arrhythmias, because of 1) changes in blood pressure, 2) acidosis induced by convulsion which increases vagal tone, 3) asphyxia which increases the irritability of heart muscle, 4) sudden change in intrathoracic pressure etc. $^{22)}$ In our experiment, central nervous stimulation caused ventricular extrasystoles of the same type as before, even after convulsion was 
eliminated by an injection of succinylcholine. Convulsion itself is not the decisive factor for arrhythmias.

Electrocardiographically, ST-T change was also often observed on central nervous stimulation. It has been investigated that the stimulation of cardiac sympathetics or infusion of catecholamines also induced ST-T change and this change was found to be not in parallel relation with change in coronary blood flow or blood pressure. It is considered that the process of electrical excitation of heart muscle is directly influenced by these neural or humoral elements. ${ }^{23), 24)}$ However, there was no evidence that the arrhythmias were always preceded by such ST-T change. There should be some intracardiac mechanism to induce the arrhythmias other than those expressed as ST-T change.

\section{SuMmary}

The change in the heart rate and arrhythmias produced by electrical stimulation of the brain stem of dogs were observed and analyzed.

The results are as follows;

(1) In the early phase of the stimulation, sinus tachycardia was mostly observed, especially marked on the stimulation of the posterior part in the hypothalamus, the midline, ventral and medial nuclei groups in the thalamus and the posterior commissure, the central gray stratum and a part of reticular formation in the midbrain. Sinus bradycardia was infrequent and not so marked.

(2) In the late phase or after the end of the stimulation, bradycardia was often observed and sometimes accompanied by such arrhythmias as atrioventricular block, nodal escaped beat or nodal escaped rhythm. This phenomenon was marked especially when blood pressure was elevated remarkably. It was explained mainly as vagal reflex, partly delayed vagal reaction of the stimulated area.

(3) Ventricular extrasystole induced by central nervous stimulation was classified into 2 groups ' $A$ ' and ' $B$ ', according to the mode of appearance. The extrasystole of type ' $A$ ' appeared in the early phase of the stimulation, independently upon elevation of the blood pressure and was perhaps of right ventricular origin. The extrasystole of type ' $B$ ', the appearance of which was similar to that of escaped beat (that is, of longer coupling), occurred in the late phase or after the cessation of the stimulation, associated with a blood pressure elevation and was thought to be of left ventricular origin. While the extrasystole of type 'A' seems to be of direct central nervous origin, the one of type ' $B$ ' is considered of secondary nature.

(4) The distribution of the area participating with the extrasystole 
of type ' $A$ ' was relatively restricted to the median portion of the brain stem. These area may have some function to influence the heart to produce ventricular extrasystoles.

\section{AGKNOWLEDGement}

The authors wish to express their thanks to Drs. I. Ito, K. Harumi, S. Mashima, A. Kuroiwa, A. Yamada, K. Shimomura and K. Ueda for their kind help.

\section{REFERENGES}

1. Levy, A. G.: Heart 4: 319, 1912-3

2. Beattie, J., Brow, G.R., and Long, C. N. H. : Proc. Roy. Soc. London 106B : 253, 1930, J.A.M.A. $95: 715,1930$.

3. Allen, W. F.: Am. J. Physiol. 98: 344, 1932.

4. Dikshit, B. B.: J. Physiol. 81 : 382, 1934.

5. van Bogaert, A.: Arch. intern. pharmacodyn. 53: 139, 1936.

6. Korth, C., Marx, H., and Weinberg, S.: Arch. exp. Pathol. \& Pharmakol. 185: 42, 1937.

7. Shinozaki, T., et al.: J. Jap. Soc. Int. Med. 27: 301, 1939, 28: 235, 1940, 29: 302, 1941 (In Japanese).

8. Weinberg, S. J. and Haley, T. J. : Circulat. Res. 3 : 103, 1955, Arch. intern. pharmacodyn. 105 : 209, 1956,- and Fuster, J. M. : Ann. Int. Med. 53 : 332, 1960.

9. Korteweg, G.C.J., Boeles, J. Th. F., and Cate, J. T.: J. Neurophysiol. 20: 100, 1957.

10. Yuasa, R., Ban, T., and Kurotsu, T.: Med. J. Osaka Univ. 8: 141, 1957, Morimoto, A. : Med. J. Osaka Univ. 4 : 69, 1951 (In Japanese), Namba, H.: Med. J. Osaka Univ. 5: 431,1954

11. Purpura, D.P., et al.: Anesthesiol, 19:27, 1958.

12. Karplus, J.P. und Kreidl, A.: Pflügers Arch. für ges. Physiol. 171 : 192, 1918.

13: Hess, W.R.: Das Zwischenhirn: Syndrome, Lokalisationen, Funktionen. Basel, Benno Schwabc \& Co. 1954.

14. Kabat, H., Magoun, H.W., and Ranson, S.W.: Arch. Neurol. \& Psychiat. 34: 931, 1935. Magoun, H.W. and Ranson, S.W.: Ergebn. Physiol. 41: 56, 1939.

15. Wang, S. C. and Ranson, S. W.: Am. J. Physiol. 132: 5, 1941.

16. Bruno, F., Guazzi, M., and Pinotti, O.: Arch. Italiennes Biol. 49:68, 1961.

17. Ueda, H., et al.: Jap. Heart J. 1: 1, 1960. Katayama, S.: Psychiatr. Neurol. Jap. $62: 357,1960$ (In Japanese).

18. Monnier, M.: Rev. neurol. 93: 267, 1955.

19. Rothberger, C.F. und Winterberg, M.: Pfügers Arch. für ges. Physiol. 132: 233, 1910.

20. Levy, A. G.: Heart 5: 299, 1914.

21. Dresel, P.E., et al. : Circulat. Res. 8: 948, 1960.

22. Hejtmancik, M.R., et al.: Am. Heart J. $37:$ 790, 1949.

23. Ikeda, M.: Jap. Circulat. J. 21 : 252, 1957.

24. Hayase, S.: Jap. Circulat. J. 21 : 228, 1957.

25. Ingram, W.R.: Handbook of Physiology; Neurophysiology II edited by Field, J., Magoun, H.W., and Hall, V.E. Amer. Physiol. Soc., Washington, D. C., p. 951, 1960. 